\title{
DEMOCRAZIA COMPETITIVA \\ E ÉLITES POLITICHE
}

\author{
di Giovanni Sartori
}

\section{Critica della critica elitistica}

Il tema che mi prefiggo di affrontare nelle pagine che seguono è, in breve, questo: le élites e le minoranze dirigenti sono un male necessario oppure, al contrario, un elemento vitale e benefico?

Una serie di autori, imponente nel tempo e per autorevolezza, si dichiara a favore della seconda soluzione. Tucidide, il massimo storico greco, ricorda che Atene raggiunse il vertice della sua grandezza con Pericle proprio perché « per rango, capacità e riconosciuta onestà egli era in grado di dominare la folla, con piena indipendenza $»^{1}$. A sua volta, Bryce ha commentato l'esperienza piú avanzata del suo tempo con questa frase lapidaria: «Nessun'altra forma di governo probabilmente ha bisogno di grandi leaders quanto la democrazia $»^{2}$. Cinquant'anni piú tardi, nel 1937, dopo il crollo della democrazia in Italia, Germania e Spagna, De Madariaga scriveva: « Nonostante le apparenze, le democrazie liberali dipendono dalla leadership forse anche piú di altre piú autoritarie forme di governo; infatti ... la loro tendenza naturale a indebolire le sorgenti stesse dell'autorità politica ha bisogno di trovare un contrappeso in un maggior grado di ... autorità dei loro leaders $»^{3}$. Nello stesso anno Mannheim giungeva alle stesse conclusioni: «la mancanza di

Il presente articolo riproduce parti del cap. VI della seconda edizione, riveduta e ampliata di Democratic Theory, di prossima pubblicazione presso la Liberty Press, Indianapolis.

${ }_{1} \mathrm{La}$ Guerra del Peloponneso, libro II, cap. VII.

2 J. Bryce, The American Commonwealth, New York, Macmillan, 1888, III, p. 337.

${ }^{3}$ S. de Madariaga, Anarchie ou Hiérarchie, Paris, 1936, p. 56. 
leadership nelle società di massa tardo-liberali va considerata come conseguenza di un cambiamento in peggio nel processo di selezione della dirigenza. Eे questa generale mancanza di direzione che apre spazi di opportunità ai gruppi con ambizioni dittatoriali $»^{4}$. Verso la fine della seconda guerra mondiale, in un testo classico degli anni '40, Lindsay dichiarava: «Se vuole sopravvivere, la democrazia dovrà sfruttare ogni briciola di abilità, di sapere e di leadership che riuscirà ad avere. Non si può dirigere il complesso mondo interdipendente in cui viviamo senza sapere e competenza, previdenza e leadership. Ogni culto dell'incompetenza non porterà che al disastro $»^{5}$. Il concetto riaffiora in V.O. Key, l'autore che piú difende la saggezza dell'elettorato: «Il punto critico per la buona salute di un ordine democratico sta nelle credenze, negli standards e nella competenza di quanti rappresentano, nell'ordine, la classe influente, i leaders d'opinione, gli attivisti politici ... Qui stanno le responsabilità quando una democrazia tende all'indecisione, alla decadenza e al crollo ... ${ }^{6}$.

Sono tutti (e si potrebbe continuare all'infinito con le citazioni) autori degni di rispetto. Inoltre, le loro parole rispecchiano con sincerità il frutto dell'esperienza, anzi di un lungo, pensoso e spesso doloroso processo di apprendimento. Eppure, quando si esamini la teoria generale della democrazia, non si può dire che il loro messaggio sia stato tradotto in termini teorici: il loro elogio della leadership resta un elemento marginale, di poco peso nella formulazione teorica. Ed è significativo che ogni tentativo di coerenza, cioè di integrazione di tale aspetto nella teoria generale, venga duramente contrastato, o addirittura denunziato, come provano ampiamente gli attuali attacchi contro «l'elitismo ${ }^{7}$. E perciò tempo di affrontare a fondo la questione.

${ }^{4} \mathrm{~K}$. Mannheim, Man and Society in an Age of Reconstruction, London, Routledge and Kegan, 1954; trad. it. L'uomo e la società in un'età di ricostruzione, Milano, Comunità, 1959, p. 84.

5 A. D. Lindsay, The Modern Democratic State, London, Oxford University Press, 1943, p. 261.

6 V. O. Key, Public Opinion and American Democracy, New York, Knopf, 1961, p. 558. La difesa dell'elettorato è The Responsible Electorate, Cambridge, (Mass.), Harvard University Press, 1966.

7 Il volumetto che ha lanciato questa etichetta è $P$. Bachrach, The Theory of Democratic Elitism: A Critique, Boston, Little Brown \& Co., 1967. La letteratura su élite e democrazia è vasta. Vedi Suzanne Keller Beyond the Ruling Class, New York, Random House, 1963; T. B. Bot- 
Ho esaminato altrove le interazioni fra inerzia dei fatti e tensione degli ideali. La mia tesi-chiave è che la democrazia ha bisogno, insieme, di « realismo » (consapevolezza dei fatti) e di «idealismo » (pressione dei valori sui fatti) ${ }^{8}$. A questo punto la domanda è: come è che questi criteri vengono applicati alla dimensione verticale della democrazia? La risposta è semplicissima: non vengono applicati affatto.

La realtà nuda e cruda è che gli « ideali » della democrazia - sovranità popolare, eguaglianza e autogoverno ${ }^{9}$ - sono rimasti piú o meno come erano nel IV secolo a.C. ${ }^{10}$; e se gli ideali della democrazia sono ancora, in linea generale, quelli greci, vuol dire che si rivolgono a una democrazia diretta, non ad una democrazia rappresentativa. Eे come dire che ancor oggi la deontologia e la pressione dei valori democratici si rivolgono soltanto alla dimensione orizzontale della politica. Certo, anche la polis greca aveva magistrati e un minimo di struttura verticale, ma il verticalismo della democrazia in una città antica sta al verticalismo di una democrazia rappresentativa su basi nazionali come la torre di un campus americano sta all'Everest. La realtà nuda $\mathrm{e}$ cruda, ma non per questo meno grave, è che abbiamo creato - con un quasi-miracolo che Rousseau ancora giudicava impossibile - una democrazia rappresentativa senza «sostegno di valore ». E non solo la costruzione verticale della democrazia su vasta scala non è stata accompagnata, nel suo divenire, da un «ideale » a lei consono, ma gli ideali a disposizione possono trasformarsi da un giorno all'altro in un grido di battaglia contro la democrazia rappresentativa, come si è constatato in questi ultimi dieci anni. Questa è, dunque, la situazione: la nostra città politica resta abbandonata all'inerzia dei fatti, e per di piú facilmente riscopre nei propri ideali degli idea-

tomore, Elites and Society, London, Routledge and Kegan, 1964, trad. it. Elite e società, Milano, Il Saggiatore, 1967; Geraint Parry, Political Elites, London, G. Allen and Unwin, 1969; trad. it. Le élites politiche, Bologna, Il Mulino, 1972, che prende in esame anche i lavori di F. Hunter, R.A. Dahl, N. Polsby e R. Presthus; A. S. McFarland, Power and Leadership in Pluralist Systems, Stanford, Stanford University Press, 1969.

${ }^{8}$ Questo, mi si permetta di ricordarlo, è il filo conduttore e la tesi ricorrente del mio libro. Vedi nota successiva.

9 Vedi la nuova edizione di Democratic Theory, cit., cap. IV, sez. 1.

10 Eccezione fatta per la «valutazione » dell'individuo. Ma è un'eccecione che non sposta in questa sede. 
li contrari. Nella migliore delle ipotesi, per non dire altro, la dimensione verticale della democrazia rimane, ad oggi, senza ideali. E se gli ideali senza fatti sono in ultima analisi «perdenti », i fatti senza la pressione degli ideali sono «perversi ». Il cerchio cosí si chiude.

Posto il problema in questi termini, è evidente che $\mathrm{i}$ cosiddetti elitisti hanno ancora una lunga via da percorrere (almeno per meritarsi davvero la loro etichetta), mentre gli anti-elitisti sono andati progressivamente - o meglio regressivamente arretrando di parecchio. La caratteristica di molta letteratura dell'ultimo decennio consiste nella pura e semplice cancellazione di tutta l'enorme testimonianza storica e di ricerca che si è accumulata fra gli ideali greci e quanto da allora è accaduto ed è stato scoperto. Il mondo reale recepito da questa letteratura non rappresenta che una particella infinitesimale - storicamente e geograficamente - delle reali complessità del mondo. Peggio ancora, si porta sempre questo mondo reale in tribunale, mai in laboratorio; lo si cita quando induce al rifiuto, e lo si ignora bellamente quando potrebbe addurre prove. In questo senso l'attacco anti-elitistico ha aperto la strada a una letteratura tutta di ideali, senza fatti: i fatti a sostegno non si presentano perché non esistono, e quelli contrari vengono tranquillamente cancellati.

Comunque sia, riprendendo il filo del mio discorso, appare chiaro che la democrazia diretta (passata o presente) non ha né bisogno né spazio per una pressione di valore « verticale». D'altra parte, tutte le piccole democrazie di tipo diretto sono inevitabilmente parte, in realtà, di piú vaste unità $e$, in definitiva, micro-componenti di un'unità totale che è sempre, e necessariamente, una democrazia indiretta legata a processi di verticalità. Dobbiamo allora abbandonare sempre piú tali processi alla loro «naturale » perversità inerziale? Correlativamente possiamo guidare, e anzi migliorare, il nostro futuro semplicemente ridando vita agli ideali del passato, cioè a ideali che, nel migliore dei casi, sono estranei alla natura e ai problemi della democrazia rappresentativa? Non sono certo questi i suggerimenti degli anti-elitisti attenti e preparati (rispetto a quelli, che non citerò, di tutta ignoranza). Se cosí accusati, risponderebbero che non è questo il loro messaggio. Eppure questi sono stati gli effetti del loro attacco, a prescindere dalle intenzioni. Siamo cosí arrivati al perno della questione. Sono convinto che il danno del messaggio emerso sul finire degli anni '60, superi 
ampiamente i vantaggi a breve termine. $E$ un messaggio che non eliminerà la verticalità; la potrà soltanto snaturare in peggio. Svalutando la meritocrazia, otteniamo solamente l'immeritocrazia; svalorizzando la selezione otteniamo solamente la disselezione; svalorizzando l'eguaglianza nel merito otteniamo solamente l'eguaglianza nel demerito. Ed è strano che una letteratura interessata soprattutto agli ideali non riesca a percepire che queste sono le conseguenze del proprio idealismo.

Come appare chiaro, non ho cercato di raddolcire i contrasti. D'altro canto, dividendo i due campi come li ho divisi, siamo in grado di capire che, se indubbiamente è in corso una battaglia, gran parte del combattimento gira ai margini del tema principale e si svolge nei punti sbagliati, con scontri periferici o addirittura inutili. Per esempio, nel concludere la sua polemica, Bachrach dice: «Se è tempo di rinunciare al mito della fedeltà dell'uomo comune alla democrazia, è anche tempo che le élites in generale e gli studiosi di politica in particolare ammettano che senza il sostegno attivo dell'uomo comune alla lunga non si può salvare la libertà. La battaglia per la libertà finirà persa in contumacia, se le élites si isolano dal popolo. Il modo migliore per garantire la sopravvivenza della democrazia consiste nell'ottenere il sostegno del popolo con uno sforzo incessante per rendere significativa la democrazia nella vita di tutti gli esseri umani ${ }^{11}$.

E strano che una simile conclusione sia presentata come un « approccio alternativo », poiché nessuno degli autori viventi messi in discussione le sarebbe contrario ${ }^{12}$. Anzi, e in linea generale, il punto ben rilevato è questo: un sistema « aperto » di minoranze in competizione non è sufficiente; la competizione può degenerare in collusione (o in pura demagogia, io aggiungerei); e la domanda senza risposta resta ancora: « aperto a chi, per che cosa? ». Comunque questa critica conferma la mia tesi, cioè che le richiamate deficienze non troveranno rimedio finché si lascerà operare la meccanica del sistema in un vuoto di pressione di valore. Ancora una volta la domanda è: come è pensabile che i processi verticali della democrazia possano funzionare senza una deontologia corrispettiva, e anzi

11 Bachrach, op. cit., p. 106.

12 Per quanto mi concerne, dicevo la stessa cosa nel mio cap. II, sez. 2, con la sola variante che «la vita» di Bachrach era, nel mio testo, «le menti ». 
con una dimensione verticale sempre piú svuotata del suo contenuto di valore?

Resta l'imputazione principale: la teoria elitistica gioca sull'equivoco tra una definizione ampia della politica e una ristretta, e finisce col rendere la politica in genere equivalente alla politica d'élite (governativa) ${ }^{13}$. Ma i critici qui sono molto piú in equivoco dei criticati. Se tutti possiamo sbagliare quando cerchiamo (e ancor piú quando non cerchiamo) di segnare i confini della politica, è semplicemente falso che Dahl, o Raymond Aron, o gli altri accusati ${ }^{14}$, sottovalutino l'importanza vitale (proprio per la politica della democrazia) delle strutture intermedie e dei gruppi e associazioni volontarie di Tocqueville in breve, del pluralismo sociale. $\mathrm{E}$ vero, invece, che la teoria competitiva della democrazia si rivolge soprattutto al sistema nel suo complesso, lo Stato democratico, e che essa rifiuta di confondere (come invece tendono a fare gli anti-elitisti) il sistema globale con i suoi sub-sistemi, cioè il macro-sistema con i microsistemi. Una volta ancora, il concetto di politica orizzontale e verticale ${ }^{15} \mathrm{ci}$ guida nel labirinto, e indica in quali punti realmente esista un contrasto d'opinioni. La caratteristica della teoria competitiva della democrazia consiste nel fatto che essa ha a che fare con la dimensione verticale. È questa la sua ragion d'essere; e ciò è tanto piú necessario, voglio aggiungere, quanto piú ci troviamo davanti alla medicina anti-elitistica di una pura e semplice "estensione orizzontale ». Non c'è, dunque, un contrasto reale quando si afferma che la democrazia politica esige

13 Secondo T.B. Bottomore, l'incoerenza di Mosca e Aron «consiste nello spostarsi, in fasi diverse del dibattito, dal concetto di una pluralità di élites al ben diverso concetto di una molteplicità di associazioni volontarie $»$; ed è giusto dire che la tesi delle associazioni volontarie fiorenti « non va a sostegno delle teorie dell'élite »; (op. cit., pp. 118-119). Similmente la tesi di Bachrach si basa, sostanzialmente, sul rifiuto di restringere la democrazia a un «metodo politico e nell'allargarne « l'ambito fino a includervi le istituzioni private piú potenti » $\mathrm{e}$, in definitiva, una partecipazione generalizzata (op. cit., p. 97).

${ }^{14} \mathrm{La}$ mia posizione sul punto è riassunta nella voce Democracy, in International Encyclopedia of The Social Sciences, New York, 1968, vol. IV, pp. 113-20. Per la posizione di Aron vedi, in particolare, Social Structure and Ruling Class, in "British Journal of Sociology », I, (1950); e Classe sociale, Classe Politique, Classe dominante, in « Archives Européennes de Sociologie », II (1960).

${ }_{15}$ Queste sono le categorie secondo le quali ho ricostruito il concetto di politica da Aristotele ad oggi (cfr. What is 'politics', in « Political Theory », I, 1973). 
sviluppo della partecipazione, democrazia sociale, democrazia industriale, e in generale micro-democrazie. Sotto questo aspetto le differenze sono una funzione della maggiore o minore importanza data da un autore ai problemi dell'applicabilità. Il contrasto vero è invece in ciò: nel concepire la dimensione orizzontale, cioè la democrazia partecipativa, non come sostegno, ma come un sostituto della democrazia rappresentativa. Comunque, dato che gli antielitisti attenti e preparati non affermano che si può fare a meno della democrazia rappresentativa, se ne può dedurre che anche la controversia su chi, a torto, restringa o allarghi il confine della politica è suscettibile di reciproci chiarimenti.

Smussati gli angoli superflui, ci troviamo al punto essenziale. Che è, in una parola, «l'eguaglianza », il modo in cui formuliamo e manovriamo questo concetto. Potremmo dire che, se l'eguaglianza è il valore centrale della democrazia «orizzontale », per ciò stesso non può essere il valore centrale della democrazia «verticale ». Dirò invece: se tutti i valori della democrazia devono derivare dall'eguaglianza, allora è questo il punto in cui non dobbiamo dimenticare che l'eguaglianza è un principio di valore, anzi un principio di valore plurivalente, di grande ricchezza. Ne viene, come avvertenza, che piú un tale principio di valore risulta mutilato, piú la democrazia ne viene impoverita e, alla fine, distrutta dal proprio « non valere ».

Rifacendosi sic et simpliciter alla lezione di Platone e Aristotele, Montesquieu scriveva che « il principio di democrazia si corrompe non solo quando si perde lo spirito dell'eguaglian$\mathrm{za}$, ma anche quando si entra in uno spirito di eguaglianza estrema, per cui ognuno vuole essere eguale e pari a coloro che ha scelto per farsi governare ». E aggiungeva: «La democrazia deve evitare due eccessi: lo spirito della diseguaglianza, che porta ... al governo di un solo individuo; e lo spirito dell'eguaglianza estremistica, che porta al dispotismo di un solo individuo ${ }^{16}$. Il suo antagonista Rousseau sembra riecheggiarne i concetti nel Discours sur l'origine de l'inegalité, quando conclude che « è contro le leggi della natura, comunque le vogliamo definire, che un imbecille guidi un saggio ». Possiamo aggiungere che, comunque noi definiamo l'imbecille e il saggio, diciamo continuamente «Tizio vale poco » o, al contrario, «Caio è di prim'ordine ». I criteri di tali giudizi hanno poca importanza: essendo gli uomini nel fatto molto diversi, ognuno

${ }^{16}$ L'Esprit des lois, libro VIII, cap. II. 
valuta «l'altro » secondo proprie pietre di paragone, e molto spesso secondo pietre di paragone subconscie e confuse. Resta il fatto che, sempre, giudichiamo gli altri e attribuiamo loro un valore in quanto possessori di qualità superiori, pari o inferiori.

La scelta, in concreto, è allora tra eguagliare verso l'alto, oppure eguagliare verso il basso. Quando si arriva a questo momento di scelta gli anti-elitisti, anche se inconsapevolmente, schiacciano l'acceleratore in pro di una parificazione verso il basso, perché la loro tesi si fonda esplicitamente sul valorizzare solamente un concetto (e una estensione) orizzontale della democrazia. Ma la tesi degli «elitisti » è davvero, per converso, l'opzione opposta, vale a dire la parificazione verso l'alto? Affronterò questo problema nella parte conclusiva. Nel frattempo debbo chiarire che il mio concetto di elite differisce da quello di altri supposti elitisti.

Una maggioranza di studiosi, specie americani, si conforma oggi ad una qualche variante della definizione di Lasswell e Kaplan: «Le élites sono formate da coloro che hanno il potere maggiore in un gruppo ${ }^{17}$. Dal mio punto di vista, si tratta di una definizione che appartiene a quell'errato realismo che toglie a un principio di valore il suo contenuto di valore ${ }^{18}$. L'intenzione di Lasswell e Kaplan è certamente di essere soltanto neutrali rispetto ai valori, ma questo tipo di semplicistica Wertfreibeit non può funzionare, e non funziona mai, come si vorrebbe. Se si definiscono le élites, e specificamente quelle politiche, « $\mathrm{i}$ detentori del potere di un corpo politico ${ }^{19}$, si equiparano qualità di élite e posizioni di potere. Di conseguenza, non possiamo piú stabilire se $\mathrm{i}$ potenti rappresentino delle élites autentiche o apocrife ${ }^{20}$. Cosí la conseguenza inevitabile della definizione tipo Lasswell è — a livello teorico ${ }^{21}$ — una

17 H. D. Lasswell e A. Kaplan, Power and Society, New Haven, Yale Univ. Press, 1950; trad. it. Potere e Società, Milano, Etas Kompass, 1969, p. 218.

18 Vedi Democratic Theory, cit., cap. III.

19 H. Lasswell e D. Lerner, The Comparative Study of Elites, Stanford, Stanford Univ. Press, 1952, p. 13.

${ }_{20}$ Questo era anche e proprio il problema di Wright Mills, che opponeva l'élite del potere all'élite intellettuale, cercando di rendere la prima responsabile verso la seconda (cfr. Wright Mills, Causes of World War Three, New York, 1958; trad. it., Le cause della terza guerra mondiale, Milano, Feltrinelli, 1959, cap. 7).

${ }^{21} \mathrm{La}$ precisazione sta per dire che qui non mi occupo della definizione operazionale. 
di queste due: o attribuire gratuitamente valore di élite a quella qualsiasi struttura di potere che di fatto esiste, o svalorizzare quel qualunque valore che può trovarsi in tale struttura di potere. Da qui si può approdare alla santificazione dello status quo o, al contrario, alla sua totale dissacrazione. Nel primo caso l'attacco anti-elitista trova qui la sua migliore giustificazione, nel secondo il suo padre naturale. In entrambi i casi ci sfugge, mi sembra, il punto cruciale.

Per tirare i remi in barca, la constatazione che le minoranzeguida esistono e probabilmente continueranno a esistere, lascia le cose esattamente come stanno. L'osservazione secondo cui «il guaio del paradiso pluralista è che i cori angelici cantano con toni marcatamente 'di ceti alti' " ${ }^{22}$ può sembrare profonda, ma contribuisce soltanto a degradare la democrazia in un sistema di invidia sociale. Per questa via la santificazione dell'esistente viene semplicemente rovesciata nella santificazione del livellamento: niente è merito, tutto è privilegio. L'alternativa costruttiva consiste nel concepire l'eguaglianza - nella dimensione verticale - come un valore « elevante »; ed è questa l'alternativa che presenterò nella conclusione. Ma prima di affrontare il problema in termini teorici, è bene inquadrarlo nel tempo e nello spazio. La domanda calzante è: dove sta, oggi, il rischio principale?

L'indicatore che coglie la concretissima e inesorabile tendenza delle nostre società è l'inflazione: l'inflazione ha mutilato le speranze di democrazia di tutto il continente latino-americano, l'inflazione rappresenta la grande angoscia di molti paesi europei, e ogni governo immancabilmente si impegna a ridurla o almeno a contenerla. Sappiamo che le cause dell'inflazione sono molto complesse, e anche di carattere internazionale. Eppure è impossibile non vedere che l'elemento innescante e fuori controllo è costituito dall'eccesso di domanda rispetto alle risorse, dall'eccesso di consumo rispetto alla produzione. Qui l'inflazione rinvia a una classe politica che troppo promette $\mathrm{e}$ concede $^{23}$. E una vicenda ben documentata da molti, se non tutti, i bilanci europei: gli stanziamenti «popolari » aumentano costantemente, mentre diminuiscono quelli impopolari - non

22 E. E. Schattschneider, The Semisovereign People, New York, Holt, Rinehart and Winston, 1960 , p. 35, citato da Bachrach, op. cit., p. 37.

${ }^{23}$ Se ne trova la prova nell'esperimento proposto da Hayek: abolendo il monopolio governativo del denaro, l'inflazione scomparirebbe im- 
solo quelli di carattere militare, ma tutti gli investimenti a rendimento non immediato ${ }^{24}$. Per riassumere una storia lunga, non si può negare che i nostri governi vanno tutti, chi piú chi meno, perdendo di autorità; che l'orizzonte temporale delle loro decisioni è diventato molto ristretto, sempre piú vicino al vivere alla giornata; e che tutti, piú o meno, sono sovraccarichi, assillati da troppe richieste che non sono in grado di soddisfare. $\mathrm{Ne}$ deriva un quadro generale di indecisione - incapacità di prendere decisioni o di portarle a effetto - miopia e inefficien$\mathrm{za}^{25}$.

Non tutto è sgradevole, in questa situazione. Anzi ciò dimostra - contro le opposte asserzioni di perfezionisti e populisti - che la democrazia rappresentativa non è affatto un'impostura, perché quanto sopra sta per un rapporto rappresentativo caratterizzato da un massimo di responsiveness. Ma tale ricettività, come tutto il resto della famiglia, soggiace alla regola del principio opposto, o del pericolo opposto ${ }^{26}$. La troppa rispondenza è già approdata alle rive della bancarotta - una realtà sempre piú frequente per i governi locali. Cosí il problema pressante è diventato quello della « responsabilità », l'altro elemento costitutivo della rappresentanza ${ }^{27}$. E piú abbiamo abbondato in sintonizzazione e ricettività, piú è necessaria una responsabilità indipendente - che è proprio il nucleo del problema della leadership.

Dove stanno, allora, i pericoli attuali e imminenti? Nella minaccia, entro le nostre democrazie, di un qualche tipo di «dominio » di minoranza? Una tale risposta, a mio parere,

mediatamente. Cfr. F. A. Hayek, Denazionalization of Money, London, Institute of Economics Affairs, 1976. Possiamo non aderire alla proposta, ma l'esercizio mentale rimane proficuo.

S. Brittan sintetizza il problema in questa formula: «la mancanza di vincoli di bilancio fra gli elettori », The Economic Contradictions of Democracy, in «British Journal of Political Science», III (1975), p. 139. Vedi anche J. M. Buchanan, R. E. Wagner, Democracy in Deficit, New York, 1977.

${ }^{24}$ Cfr. R. Rose e B. Peters, Can Government Go Bankrupt? A Preliminary Inquiry into Political Overload, di prossima pubblicazione.

${ }^{25}$ Cfr. M. J. Crozier, S. P. Huntington, J. Watanuki, The Crisis of Democracy: Report on the Governability of Democracies to the Trilateral Commission, New York, New York Univ. Press, 1975, trad. it. La crisi della democrazia, Milano, Franco Angeli, 1977.

${ }^{26}$ Vedi Democratic Theory, cit., cap. IV, sez. 5.

27 Vedi la mia voce, Representational Systems, in International Encyclopedia of The Social Sciences, cit., vol. XIII, pp. 465-74. 
sfida ogni comprensione intelligente di quanto è realmente in gioco: è una fuga in un altro mondo. Quanto piú cresce la complessità dei problemi, tanto piú ci viene proposta la piú puerile delle soluzioni: sarà il popolo a risolverli. L'idea sottostante è che la traiettoria della democrazia è stata fissata una volta per tutte; che se e quando si presentino smentite o fatti non voluti, vanno ignorati; e che, in sostanza, dovremmo combattere sempre la stessa battaglia della prima guerra. Io vedo, sí, minacciose minoranze di ogni genere ammassarsi all'orizzonte - ma per il prossimo atto. Nel frattempo, il mio timore è che le nostre democrazie possano naufragare sugli scogli della bancarotta, del sovraccarico e della ingovernabilità, creando cosí i presupposti per sostituire alle minoranze «che si propongono » una minoranza che «si impone ${ }^{28}$. Temo, allora, proprio quel che gli anti-elitisti apparentemente sperano, o nei fatti promuovono: che la democrazia possa in definitiva divorare - come Saturno i suoi figli - i propri leaders.

\section{La legge di ferro dell'oligarchia}

Resta ancora un'obiezione di fondo - «la legge ferrea dell'oligarchia » di Michels - che pone in dubbio la possibilità stessa di democrazia, mettendoci cosí davanti al problema preliminare di come e dove cercare la democrazia. E vero che Michels non proponeva una teoria generale della democrazia, ma concentrava la sua attenzione sul partito politico. Il titolo originale della sua opera piú importante, scritta nel 1910, era Sociologia del partito politico ${ }^{29}$. Tuttavia le conclusioni che si possono trarre dalla sua analisi sono indubbiamente di importanza cruciale per l'intero problema della democrazia, e per due ragioni.

La prima è che un sistema democratico è, operativamente, un sistema partitico, come dice Kelsen: « La democrazia moderna si fonda totalmente sui partiti politici; piú è vasta l'applicazione del principio democratico, piú sono importanti i parti-

28 E l'indovinata formulazione dell'antitesi fra democrazia e non, di F. Burzio, Essenza e Attualità del Liberalismo, Torino, UTET, 1945, p. 19.

${ }^{29}$ Il libro uscí in Germania nel 1911 e in Italia nel 1912; essendo Michels bilingue, si possono considerare originali entrambe le edizioni. Si veda ora nell'edizione del Mulino, Bologna, 1966. 
ti ${ }^{30}$. In realtà, i partiti politici sono divenuti un elemento cosí essenziale che molti autori vedono nella democrazia non semplicemente un sistema partitico, ma una partitocrazia, intendendo con ciò che il centro del potere è passato di fatto dal governo e dal parlamento alle direzioni dei partiti ${ }^{31}$. E non è tutto. La fenomenologia dei partiti ha un'importanza paradigmatica. Se, infatti, la vita democratica nasce dalla creazione volontaria di piccole e libere comunità inter pares, anche i partiti si formano come associazioni volontarie e di tali associazioni rappresentano l'espressione politica tipica in un sistema democratico su vasta scala. Da questo punto di vista, dunque, $\mathrm{i}$ partiti diventano il tipo di organismo politico che piú assomiglia, o dovrebbe assomigliare, al prototipo di qualsiasi autentica democrazia politica.

Senza dubbio Michels mette il dito su un punto strategico. In piú, e ancor piú significativamente, esamina il problema dell'organizzazione, e oggi non c'è campo dell'attività umana che non cerchi di allargare e perfezionare la propria organizzazione. Anzi «l'organizzazione» costituisce un elemento e una dimensione cruciali della nostra vita. Perciò, sotto tutti gli aspetti, non dobbiamo sottovalutare il peso della conclusione di Michels secondo la quale l'organizzazione distrugge la democrazia e la trasforma in oligarchia: "Chi dice organizzazione, dice tendenza all'oligarchia ... Il meccanismo dell'organizzazione ... inverte completamente la posizione del condottiero rispetto alla massa ... Ovunque l'organizzazione è piú forte, si constata un minor grado di democrazia applicata $\gg 32$.

Secondo Michels questa è una «legge ferrea », un processo che non si può evitare né fermare. Ė inevitabile che ogni partito cerchi il massimo possibile di iscritti ed è quindi inevitabile che i « partiti d'opinione » si trasformino gradatamente in «partiti

${ }^{30} \mathrm{H}$. Kelsen, Von Wesen und Wert der Demokratie, cap. II. La democrazia come sistema di partiti è esaminata ampiamente nel mio libro Parties and Party Systems, Cambridge, Cambridge Univ. Press, 1976.

31 Vedi per tutti G. Maranini, Miti e realtà della democrazia, Milano, Comunità, 1958.

32 La sociologia del partito politico, cit., p. 33. Per un breve sunto delle tesi di Michels «sulle tendenze oligarchiche degli aggregati politici», vedi il suo Studi sulla democrazia e sull'autorità, Firenze, La Nuova Italia, 1933, pagg. 58-59, e il seguente passo, scritto nel 1909: «Se esiste una legge sociologica seguita dai partiti politici ... riducendola alla formula piú concisa, deve suonare cosí: l'organizzazione è madre del predominio degli eletti sugli elettori », (ivi, p. 49). 
di organizzazione ». E poiché con l'aumentata necessità di organizzazione aumenta il potere del leader, ogni organizzazione di partito tende a diventare oligarchica. Michels concludeva il suo classico testo con questa affermazione: «L'esistenza di capi è un fenomeno congenito a qualsiasi forma di vita sociale. Non incombe alla scienza investigare se sia un bene o un male ... $\mathrm{Ma}$ è di grande valore, sia scientifico che pratico, stabilire che ogni sistema di capi è incompatibile con i postulati piú essenziali della democrazia ${ }^{33}$.

Si possono muovere molte critiche alla diagnosi di Michels. In primo luogo, tale autore parla di oligarchia e di organizzazione senza mai dare una definizione precisa dei due concetti. In questo riferimento ho sottolineato altrove che Michels ha fornito, forse, una legge ferrea della burocrazia, ma soltanto una « legge bronzea » (non certo a prova di ferro) dell'oligarchia ${ }^{34}$. In ogni caso, il nocciolo della critica è questo: dato che esistono molti e diversi tipi di organizzazione, non possiamo concludere, senza specificazioni e qualificazioni, che tutti sono sempre e necessariamente oligarchie incompatibili con la democrazia. In secondo luogo, il campo di osservazione di Michels è troppo limitato, poiché in sostanza si circoscrive al partito social-democratico tedesco. In terzo luogo, dalla premessa « i partiti non sono democratici » non si può passare alla conclusione «la democrazia non è democratica ». La prova addotta è troppo esigua rispetto all'ampiezza della conclusione.

Nonostante tutto, la legge di Michels in complesso ancora tiene, anche se soltanto come «legge bronzea ${ }^{35}$. Si può infatti

33 La sociologia del partito politico, cit., p. 419. Il testo tedesco dice Fübrertum e quello italiano sistema di capi: quindi la semplice traduzione in «leadership » (come nella edizione inglese) non dà il significato dell'originale.

34 Vedi G. Sartori, Democrazia, burocrazia e oligarchia nei partiti, in « Rassegna italiana di sociologia », vol. III (1960), pp. 119-36, dove indico la bibliografia e rilevo la differenza fra le posizioni di Michels e di Max Weber.

35 Come autorevolmente confermato, fra gli altri, da Duverger: nell'opera di Michels «sono descritte in termini sempre attuali le tendenze oligarchiche delle organizzazioni di massa ». (Les parties politiques, Paris, trad. it. I partiti politici, Milano, Comunità, 1961, p. 12); e da S. M. Lipset: «Le ovvie conclusioni tratte da questa analisi sono che $\mathrm{i}$ requisiti funzionali della democrazia per lo piú non si incontrano nella maggior parte dei sindacati » (The Political Process in Trade Unions, in Political Man, New York, Doubleday, 1960, trad. it. L'uomo e la politica, Milano, 
replicare alla prima obiezione osservando che la tesi di fondo circa l'organizzazione è una generalizzazione che, per quanto vaga, si riferisce, però, a una tendenza continua e persistente. Alla seconda obiezione si può rispondere che il caso presentato da Michels, il partito socialdemocratico tedesco ante 1914, è sempre pertinente ai grandi partiti di massa europei, per origine e forma poco piú democratici della socialdemocrazia tedesca. Alla terza obiezione si è data una risposta osservando che, se si allarga l'esame a tutti i settori organizzati dell'attività politica, compresi in particolare $i$ sindacati, probabilmente non troveremo nelle altre organizzazioni maggiore democrazia che nei partiti politici di Michels; e, in questo caso, la conclusione « la democrazia porta all'oligarchia ${ }^{36}$ regge, o sembra reggere.

Com'è evidente, non sottovaluto affatto la tesi di Michels. Tuttavia la considero l'esempio « esemplare » di come si possa cercare la democrazia senza mai trovarla. Se acconsentiamo a misurare la democrazia confrontando i suoi aspetti organizzativi con il prototipo delle associazioni volontarie, è difficile dimostrare che Michels sbaglia. Ma, ecco il punto, possiamo passare da una democrazia faccia-a-faccia a una democrazia su scala di diecine di milioni, come se fossero paragonabili e appartenessero allo stesso continuo? Michels concepisce la democrazia alla Rousseau ${ }^{37}$ e al modo dei critici democratici delle nostre democrazie. Nel formulare il problema egli non differisce da Proudhon, Marx e Bakunin: tutti si richiamano alla matrice delle associazioni volontarie, e secondo questo metro concludono che nella democrazia politica in cui viviamo non esiste nessuna forma organizzata che corrisponda a quel modello.

A questo punto, le profezie si scontrano una con l'altra. Per un verso, la democrazia trionferà in futuro, ma la sua vittoria è rinviata al giorno in cui tutte le sovrastrutture organizzative che la reprimono - prima fra tutte lo Stato - saranno state distrutte. Per un altro verso, le sovrastrutture sono destinate a crescere, e quindi la democrazia resterà per sempre irrealizzabile. Nel primo caso riteniamo possibile allargare all'infinito il prototipo dell'associazione volontaria e trasformarlo nella

Comunità, 1963, p. 423). Michels viene spesso convalidato, involontariamente o indirettamente.

${ }^{36} \mathrm{La}$ Sociologia del partito politico, cit., prefazione, p. XIII.

37 Per esempio Michels dichiara che il sistema rappresentativo è impossibile, richiamandosi al postulato rousseauiano secondo cui l'esercizio della volontà non può essere alienato (op. cit., p. 37). 
gigantesca entità collettiva auto-gestita che sognavano Marx e gli anarchici. Nel secondo caso riconosciamo che nel processo di crescita il prototipo si distorce e concludiamo, perciò, che la democrazia su vasta scala è una pura utopia. Ma anche se le profezie sono in contrasto per l'avvenire, la loro premessa, per quanto riguarda il presente, è la stessa: le nostre cosiddette democrazie sono apocrife.

Sotto il profilo pratico le due concezioni si rafforzano reciprocamente nella medesima negazione: sia Michels sia i perfezionisti, il pessimista e gli ottimisti, vanno cercando la democrazia con la stessa lanterna. E il guaio sta proprio nella lanterna. Che essa dia poca luce lo rivela con evidenza il fatto che non illumina la differenza fra le nostre democrazie, dichiarate false, e le reali non-democrazie. Né Marx né Michels scorgono questa differenza, e tanto meno la spiegano. $E$ non sono in grado di spiegarla perché non hanno mai afferrato in quale modo si crei nella realtà una democrazia su vasta scala. Il loro errore consiste in un confronto senza somiglianza, e cioè nel fare un confronto con un termine di paragone irripetibile. Essi cercano la democrazia nelle strutture e non nelle interazioni, vogliono trovarla immobilizzata in qualcosa, all'interno di qualcosa, invece di cercarla nelle interazioni tra gruppi e organizzazioni. Le strutture sono importanti, senza alcun dubbio; ma la loro importanza, rispetto al formarsi di una macro-democrazia, nasce dalla dinamica dei loro rapporti reciproci. Se questo punto ci sfugge, allora approderemo sempre dove la democrazia è morta $\mathrm{o}$ non può esistere, e non arriveremo mai là dove è viva $\mathrm{e}$ vitale.

Michels cercava la democrazia dentro la grande organizzazione. Ma, dopotutto, un'organizzazione è la risposta a una « grandezza », a qualcosa che altrimenti sfuggirebbe di mano; e piú l'organismo è vasto, piú richiede strutture ben definite e di tipo gerarchico. Dunque, ricorriamo all'organizzazione per creare non una forma democratica, ma un organismo innanzi tutto ordinato e efficiente - il che è cosa completamente diversa. Quindi il nostro problema comincia dove Michels lo abbandona. Invece di esaminare l'interno di un'organizzazione, osserviamo, nel mondo della politica, i rapporti tra organizzazioni separate e in competizione.

Perché sono in competizione? Ovviamente perché cercano alleati all'esterno, dato che la loro forza deriva dal numero dei seguaci. $\mathrm{E}$ in quale modo competono tra loro? Promettendo 
- anche ciò è ovvio - benefici e vantaggi ai loro seguaci. $\mathrm{Ne}$ consegue che la maggioranza non organizzata dei politicamente inattivi diviene arbitra della gara fra le minoranze organizzate dei politicamente attivi. E cosí, per quanto oligarchica sia l'organizzazione delle singole minoranze vista al suo interno, il risultato della loro competizione è la democrazia. Detto in maniera piú incisiva: la democrazia rappresentativa deriva dal fatto che il potere di scegliere fra i competitori è nelle mani del demos.

E questo che non solo Michels, ma $\mathrm{i}$ marxisti in genere e parte degli attuali anti-elitisti, ancora non riescono a capire. Fu Schumpeter il primo a rendersene conto meglio di ogni altro. Con questo arriviamo alla teoria competitiva della democrazia, il nostro prossimo argomento.

\section{La teoria competitiva}

Secondo Schumpeter la democrazia politica è, in sostanza, un metodo o una procedura, mediante la quale alcune persone vengono scelte a guidare la comunità politica tramite una competizione che ne sancisca l'autorità ${ }^{38}$. Mettendo assieme la definizione di Schumpeter con la «legge delle reazioni previste » di Friedrich ${ }^{39}$, la definizione competitiva di democrazia può essere sviluppata come segue: la democrazia è il prodotto, o la conseguenza, degli effetti secondari e composti derivanti dall'adozione di questo metodo.

Spiegherò e approfondirò, man mano che si procede, questa definizione. Il punto preliminare è che ciò che stiamo definendo è la democrazia politica, non i processi di conflitto e di mutamento sociale ${ }^{40}$. E il punto importante è che la nostra definizio-

38 Vedi J. A. Schumpeter, Capitalism, Socialism and Democracy, London, Allen \& Unwin, 1947, tr. it. Capitalismo, socialismo e democrazia, Milano, Etas Kompass, $1973^{2}$, p. 257. Bisogna comunque leggerne per intero il cap. XXII.

${ }^{39}$ L'espressione migliore di questa formula si trova nella $2^{\mathrm{a}}$ edizione del suo Constitutional Government and Democracy, Boston, 1941, cap. XXV. Nell'edizione del 1950 il capitolo è stato omesso, benché la regola ricompaia nel successivo Man and bis Government, New York, McGraw Hill, 1963.

${ }^{40}$ Pertanto che una società sia integrata, conflittuale o basata su divisioni di classe è irrilevante. Mi sfugge, in particolare, perché la teoria del conflitto sociale di Dahrendorf (Classes and Class Conflict in the 
ne stabilisce, spiegando come funziona, che la democrazia su vasta scala non è la proiezione statica, o il semplice assommarsi, di molte piccole democrazie. Il termine di confronto, o di misura, non è piú il piccolo raggruppamento volontario, ma è un sistema di reazioni a catena. E questa è la teoria nuova della democrazia, cioè quanto vi è di nuovo nella nostra teoria. Chi non $\mathrm{fa} i$ conti con il modello delle reazioni a catena o ha poco di nuovo da dire, o non può che dirlo in maniera sbagliata - senza prospettiva, appunto, di «modello ».

Parlare di modello è esatto, perché la teoria competitiva della democrazia possiede il potere esplicativo che giustifica tale qualifica. D'altra parte, un modello non è mai una chiave onnispiegante, per lo meno nelle scienze sociali. Se taluno asserisse che l'intero processo democratico si riduce a una competizione tra élites, il nostro modello non autorizza tale pretesa. Analogamente, e piú specificamente, la teoria competitiva della democrazia non può sottintendere, e infatti non lo sottintende, che la ćemocrazia che abbiamo è la migliore che possiamo avere: il modello non riguarda la perfettibilità della democrazia. È ovvio, dunque, che la teoria competitiva della democrazia non rappresenta tutta la teoria, per intero. Però le fornisce condizioni necessarie e sufficienti perché una democrazia politica esista. E dunque esatto dire che la teoria competitiva definisce la democrazia «al suo minimo ». Resta il fatto che ora possediamo un modello capace di spiegare quanto finora è rimasto inesplicato, o quanto è stato altrimenti frainteso.

Schumpeter propose la sua teoria come «un'altra teoria». È giusto - salvo che è facile inflazionare un'altra teoria, tramutandola in una teoria alternativa. Difatti oggi è opinione diffusa che possediamo due teorie alternative della democrazia quella classica e quella competitiva - fra cui siamo liberi di scegliere, o addirittura ci viene chiesto di scegliere. Come appare dalle mie precedenti osservazioni, e come ora metterò in chiaro, si tratta di una ottica e di una opzione sbagliate. La teoria classica della democrazia riguarda, come è tenuta a fare,

Industrial Society, London, Routledge and Kegan, 1959, trad. it. Classi $e$ conflitto di classe nelle società industriali, Bari, Laterza, 1963) debba essere interpretata come un attacco alla teoria competitiva della democrazia. In realtà Dahrendorf critica Mosca, Pareto e Aron (oltre a Talcott Parsons) per la loro interpretazione delle interazioni e delle strutture sociali. 
le fondamenta ${ }^{41}$. Perciò la sua attenzione, dal punto di vista empirico, si concentra quasi esclusivamente sul singolo elettore e sul suo aggregarsi ad altri formando delle maggioranze. Correlativamente, dal punto di vista teorico, l'assunto è che una forma di governo democratica è una forma di governo controllata dalla volontà della maggioranza. Non c'è niente in questa teoria che l'«altra » sia costretta a rifiutare. Senza dubbio, le idee nuove si affermano sempre polemicamente: noi pensiamo contro. $\mathrm{Ma}$, una volta finiti il caldo e il polverone della battaglia, avvertiamo che le obiezioni non sono necessariamente negazioni. In verità, la teoria competitiva rifiuta solo la tesi del mandato, vale a dire il concetto medievale di rappresentanza ${ }^{42}$. Il piú del resto è costituito da obiezioni che vertono sui vuoti o sulle ingenuità della teoria classica. Per esempio, quando la teoria classica afferma che il potere politico, se attribuito nel modo giusto, sarebbe eo ipso benefico, formula un pio desiderio. L'obiezione principale è, allora, che le basi non rappresentano l'edificio per intero.

Tale incompletezza viene sottolineata, nella mia analisi, come un eccesso di prescrizioni in vece di descrizioni: quando la teoria classica si trova in difficoltà, ricorre al dovrebbe. In alcuni punti cruciali, gli ideali o vengono esposti in un vuoto di fatti, o vengono contrabbandati come fatti; per cui risulta incomprensibile, su tali basi, come una democrazia possa in alcun modo funzionare. E chiaro che la teoria classica si ferma prima del traguardo: non arriva a cogliere la parte, piú che essenziale, svolta dai meccanismi del sistema, e precisamente dal fatto che i suoi operatori sono obbligati a competere al cospetto di un mercato dei consumatori. E qui sovviene, con le sue integrazioni, la teoria competitiva.

Su quali basi, dunque, persiste l'idea che abbiamo a disposizione due teorie contrarie e alternative della democrazia? Secondo la mia analisi, tale idea è oggi accettata a causa di una ricostruzione genealogica che è in gran parte un artificio polemico brandito dagli anti-elitisti. In base a siffatto pedigree la

${ }^{41}$ Carole Pateman è nel giusto quando rileva che Schumpeter non è chiaro nella sua interpretazione della «teoria classica »; ma essa va oltre il segno quando afferma che "la nozione di una 'teoria classica della democrazia' è un mito ». (C. Pateman, Participation and Democratic Theory, Cambridge, Cambridge University Press, 1970, p. 17).

42 In realtà è anche dubbio che la teoria classica abbia mai incorporato la nozione di mandato, che non appartiene, essendo medievale, né alla democrazia degli antichi né alla democrazia dei moderni. 
teoria competitiva è elitistica, e in quanto tale deriva da Mosca e Pareto, o ad essi si collega ${ }^{43}$. Un'altra versione, egualmente artificiosa, è questa: che l'elemento centrale della teoria classica della democrazia è la partecipazione, come è in particolare attestato da Rousseau, James e John Stuart Mill, e G.D.H. Cole; mentre i tratti caratteristici della teoria elitista della democrazia sono la diffidenza nei confronti della partecipazione, l'elogio dell'apatia, e la fiducia nella élite ${ }^{44}$. Anche questo quadro non sta assieme. Primo, se la partecipazione è la nota dominante, allora la teoria classica della democrazia ha inizio con la democrazia greca; e la differenza che merita di essere meditata è che la partecipazione non era, per i greci, un ideale ma la loro pratica quotidiana. Secondo, Rousseau rappresenta l'ultimo esponente della democrazia pre-liberale, mentre John Stuart Mill è uno dei maggiori teorici della democrazia liberale (rappresentativa); cosí, voler trovare una continuità tra $i$ due richiede distorsioni eroiche. Terzo, e in particolare, la posizione di J.S. Mill è male interpretata sia storicamente che sostantivamente. Quando Mill scriveva che « la sola forma di governo che può soddisfare in pieno tutte le esigenze è quella in cui tutto il popolo è chiamato a partecipare ${ }^{45}$, il suffragio in Inghilterra era ancora molto ristretto, e questa affermazione era il modo piú comune a quel tempo di sostenere il suffragio universale. D'altra parte, J.S. Mill ha sostenuto anche, e molto piú vigorosamente, che uomini e donne debbano godere dei diritti politici « non perché possano governare, ma perché non possano essere mal governati ${ }^{46}$. Pertanto, qualunque corretto raffronto

43 Poiché l'ulteriore sottinteso potrebbe essere che Mosca e Pareto vennero messi a frutto dal fascismo, è bene stabilire che questo è semplicemente falso. Come rileva giustamente N. Bobbio: « Nei due dottrinari e principali artefici della dottrina del fascismo, il filosofo Gentile e il giurista Rocco, la teoria delle élites non ebbe alcuna parte, neppure di secondo ordine ... La teoria della classe politica ebbe fortuna non già tra gli scrittori fascisti ma tra quelli antifascisti e democratici ... L'unico tentativo serio ... di riprendere e approfondire le dee di Mosca ... fu quello del gobettiano democratico-radicale Guido Dorso, e l'unica rielaborazione delle idee di Pareto ... fu quella del paretiano liberale e democratico Filippo Burzio». (Saggi sulla scienza politica in Italia, Bari, Laterza, 1969, pp. 247-48).

$44 \mathrm{Si}$ fa riferimento in particolare a G. Duncan, S. Lukes, The New Democracy, in "Political Studies», II (1963) e a C. Pateman, op. cit.

45 Questa è la citazione sulla quale Duncan e Lukes, che considerano Mill il 'principale teorico della democrazia', basano la loro argomentazione; (op. cit., p. 158).

46 J. S. Mill, Representative Government, New York, 1951, p. 391. 
diacronico induce a concludere che la differenza piú significativa tra J.S. Mill e gli attuali supposti nemici della partecipazione è che egli poteva coltivare speranze che da allora hanno dovuto fare i conti con la realtà. Quarto, la posizione in tema di partecipazione e apatia dei cosiddetti elitisti è molto differenziata, e il suo spirito potrebbe difficilmente essere qualificato come uno stato di «diffidenza» - se mai, il denominatore comune sarebbe «delusione ». Quinto, la fiducia nelle élites è soltanto, come tale, l'altra faccia della ricorrente « accusa » che imputa alle élites i fallimenti della democrazia. Pertanto, l'aspetto distintivo della teoria competitiva della democrazia risiede nella sua capacità di spiegare a quali condizioni ci si può fidare delle élites e servirsene a fini democratici; e se non si afferra questo punto - come è il caso - tutto il resto è frainteso.

Quanto sopra può essere ribadito osservando che ciò che non viene mai chiesto o chiarito dagli anti-elitisti è il criterio in base al quale un « elitista » è dichiarato tale. Se questo criterio consiste nell'affermazione che il potere è inevitabilmente distribuito in modo asimmetrico e concentrato nelle mani di minoranze (non delle maggioranze), allora non è chiaro perché, per esempio, Dahrendorf e C. Wright Mills dovrebbero essere considerati anti-elitisti. Se, invece, il criterio è dato dall'intenzione, e precisamente dall'intento di dimostrare la imperseguibilità della democrazia, allora Pareto, Mosca e Michels erano degli anti-elitisti, mentre la linea che va da Schumpeter a Dahl è anti-elitista, visto che è caratterizzata dall'intento opposto. Allora, il criterio è la partecipazione? In questo caso il confine non è quanta partecipazione sia possibile avere (una questione empirica), ma se la democrazia partecipata può sostituire, a livello della polis, la democrazia rappresentativa. E in base a questo metro anche Bachrach e Carole Pateman sarebbero degli elitisti. Apparentemente non resta che il criterio di come un autore affronta il problema delle «masse » (nella relazione élite-massa). Ad esempio, si suppone che un elitista consideri «la principale funzione dell'élite quella di tenere a bada le masse, trattenendole dalle tentazioni ... del perfezionismo e dalle trappole della demagogia »; che assuma che le masse siano «degenerate »; che «lo scopo dell'elezione non è di rafforzare la democrazia », e che «il correttivo non sta nell'educare l'elettorato nel tentativo di inculcare standards piú elevati che portino alla selezione di leaders piú qualifi- 
cati $\gg{ }^{47}$. Ma chi è, oggi, un cosí perverso elitista ${ }^{48}$ ? Dato che non ne trovo, restiamo, alla fine, senza casi.

Il fatto che la genealogia che dovrebbe identificare gli « elitisti » contemporanei sia sbagliata e che il mucchio, appena analizzato, si dissolve, chiude la questione della plausibilità intellettuale della teoria delle « due teorie ». Ne resta la grandissima comodità. In verità, finché abbiamo il diritto a una scelta, la «nuova teoria» (quella competitiva) si può eliminare in un battibaleno con una sola frase: fra le due, io scelgo la teoria classica. E questo è il modo con il quale i nuovi populisti riescono a veleggiare sulle loro facili acque.

Concludendo sul punto, la questione se esistano o no due teorie della democrazia che si escludono a vicenda, esige una presa di posizione. La mia è che nella sua formulazione attuale la teoria competitiva sia né piú né meno che il completamento - nelle sue lacune - e la correzione - nei suoi squilibri della teoria classica. Ritengo anche che, per quanto riguarda il persistere dell'opinione contraria, il rimprovero dovrebbe venire ormai capovolto: mentre i denigrati elitisti aggiungono alla teoria classica, questa, in quanto tale, cioè esibita come contro-teoria, consiste semplicemente nell'ignorare l'aggiunta. Ma per dare maggiori sostegni a questa conclusione, dobbiamo lasciare Schumpeter e giungere alla teoria della « poliarchia » di Dahl ${ }^{49}$.

47 Bachrach, op. cit., pp. 40-41.

48 In realtà, Bachrach attribuisce queste opinioni a me. La deformazione e la caricatura sono cosí evidenti che il curatore della traduzione italiana di Bachrach richiama queste pagine del suo autore come un caso patente di distorsione polemica. Cfr. M. Stoppino, Presentazio$n e$, in La Teoria dell'elitismo democratico, Napoli, Guida, 1974, pp. XVII-XVIII. Ad esempio, secondo Bachrach sarei favorevole alla rappresentanza proporzionale sulla base dei seguenti argomenti: «In aggiunta alla sua superiorità nel selezionare una migliore leadership, Sartori sostiene che la rappresentanza proporzionale è un sistema migliore anche perché ... comporta sempre governi di coalizione, il che rende piú difficile per l'elettorato identificare le responsabilità politichè ». (Bachrach, $o p$. cit., p. 42). Non solo non ho mai difeso la rappresentanza proporzionale, né ho mai detto che porta a una leadership migliore (come e perché dovrebbe farlo?), ma ho detto, e ripeto ora, l'esatto contrario di ciò che Bachrach inventa: il fatto che $\mathrm{i}$ governi di coalizione non consentono all'elettorato di identificare le responsabilità è un difetto della rappresentanza proporzionale.

${ }_{49}$ Forse devo spiegare perché An Economic Theory of Democracy di A. Downs (New York, Harper and Row, 1957) non sia stata appaiata, qui, all'opera di Schumpeter. La ragione è che la teoria competitiva si richiama a un'analogia economica centrale, ma non è una 
Dato che riferirò il pensiero di Dahl con parole mie, devo avvertire il lettore di una differenza nella strategia. La strategia fondamentale di Dahl consiste nel riservare la parola democrazia al « sistema ideale » e usare « poliarchia » per il mondo reale ${ }^{50}$. Io invece accetto «democrazia » anche per il mondo reale, ma ne divido in due metà il significato: quella prescrittivo (normativo) e quella descrittivo (denotativo) ${ }^{51}$. Sono ovviamente strade parallele per aftrontare un medesimo problema; però le strategie implicano differenze. All'inizio la mia linea d'analisi non comporta grandi scoperte, ma semplicemente rileva, per esempio, che la democrazia come « potere del popolo » è una concezione normativa, mentre la democrazia come «poliarchia » è una concezione descrittiva. Tuttavia ora l'attenzione è concentrata sistematicamente su come gli ideali influiscano sulla realtà e, viceversa, su come il mondo reale accolga, ma anche vanifichi e inganni, gli ideali. In breve, seguendo questa via ci si sofferma sulla gestione degli ideali; e la scoperta è che, in ordine a tale gestione, siamo ancora nell'età infantile. Tutto bene (si spera) fino a quando giungo al nodo finale: definire la democrazia lungo la dimensione verticale. Poiché mi incombe di farlo sia descrittivamente che normativamente, è proprio a questo punto che la mia strategia mi crea dei problemi, e mi mette nei guai.

Dal punto di vista descrittivo, il « modello delle reazioni a catena » si può rendere dicendo che la democrazia è una poliarchia elettorale. In Dahl questa etichetta sarebbe ridondante, perché la sua concezione di poliarchia comprende, per definizione, elezioni libere e competitive (insieme ad altre proprietà). Senonché quando le etichette hanno successo acquistano vita propria, e il loro peso semantico prevale sulla concettualizzazione del loro inventore. Ora, semanticamente, « poliarchia » è il contrario di « oligarchia »; e quindi il termine poliarchia indica soltanto, in sé e per sé, che un'oligarchia si è spezzata, che si è trasformata in una costellazione multipla e diffusa di gruppi di potere (né coerente né omogenea) che sono, al massimo, aperti. Se risultano aggiunte altre proprietà, si tratta di

«teoria economica». Mi sono occupato dell'importanza dell'analisi di Downs in Parties and Party Systems, cit., cap. 10.

50 Cfr. Polyarcby, New Haven, Yale University Press, 1971, p. 9. Le poliarchie vengono qui definite come « regimi relativamente (ma non completamente) democratizzati ».

51 Vedi Democratic Theory, cit., cap. I, sez. 1. 
extra, anzi di elementi estranei, che il termine - nella sua trasmissione - lascia inevitabilmente cadere. In questa ottica, dunque, "poliarchia elettorale » non comporta una ridondanza, come dimostra il fatto che è perfettamente concepibile una poliarchia non-elettiva. Il mondo medievale, ad esempio, potrebbe rientrare in una descrizione poliarchica, salvo che per una proprietà: l'apertura. Comunque, l'essere aperta non è semanticamente una proprietà o caratteristica indispensabile della poliarchia. Il concetto di apertura si associa alla poliarchia perché, anche se non lo diciamo, noi intendiamo poliarchia « elettiva »: infatti è il ricorrere sistematico delle elezioni che comporta l'apertura del sistema.

In ogni modo, un sistema politico può essere poliarchico (nel senso semantico) senza fondarsi sul suffragio popolare. Anche in questo caso sarà molto diverso da un'oligarchia, e anche da un'oligopolio, a causa della sua diffusione: a ogni unità di potere resterà un potere piccolo (in estensione) o scarso (in intensità). Perciò una poliarchia non-elettiva comporterà, in misura significativa, una reciproca delimitazione e controllo fra i leaders, almeno nel senso che la sua dispersione ostacola una coagulazione in blocchi. $\mathrm{Ma}$ anche cosí siamo ancora lontanissimi dalla democrazia. Le democrazie si valgono anche esse del reciproco controllo fra i leaders, ma solo dopo aver stabilito il controllo dei leaders, sui leaders. Il punto cruciale è quindi questo: affinché il demos possa limitare, controllare e influenzare i leaders, occorre che esso possieda, integrale e senza restrizioni, il potere di sceglierli: cioè, devono svolgersi a intervalli regolari elezioni regolari. Inoltre, cosa altrettanto importante, è l'elezione che stabilisce il legame con la competizione e con la competitività. In sé e per sé (semanticamente) né oligarchia né poliarchia implicano « competizione », almeno nel senso in cui questa dà significato alla teoria competitiva della democrazia. Dunque, poliarchia elettorale. Non conviene abbreviare ulteriormente questa sintesi.

La definizione suindicata è solo descrittiva - e cioè solo la parte descrittiva della mia indagine definitoria - ma è anche, come ho già precisato, una definizione « minima ». Non riguarda la perfettibilità, ma la fattibilità della democrazia. Pure, prima di addentrarci nella « massimizzazione », assicuriamoci di non perdere di vista quanto la nostra definizione minima già contiene.

Chiamando democrazia una poliarchia, non affermiamo sem- 
plicemente che piú leaders prendono il posto di uno solo. Se la differenza fosse tutta qui, non ci sarebbe da stare molto allegri. In pari tempo, parlando di poliarchia elettiva, non si vuole sostenere che ci è soltanto consentito di scegliere fra diversi possibili leaders. Se fosse tutto qui, si potrebbe osservare, in vena di rifiuto, che i leaders cambiano, ma il dominio resta. Tuttavia non è cosí, e anzi una simile considerazione proverebbe che non si è capito il modello della reazione a catena né, specificamente, il ruolo centrale che in questo modello gioca la legge delle reazioni previste. E questa legge che collega e sintonizza il momento elettorale con $\mathrm{i}$ periodi interelettorali. Se è vero - come è vero - che il leader soggetto alla prova elettorale periodica è costantemente preoccupato per come gli elettori reagiranno alle sue azioni, ne consegue che egli sarà costantemente pilotato dall' « anticipazione » di quella possibile reazione, negativa o positiva. Con Friedrich, dunque, il modello della reazione a catena diviene del tutto compiuto ed autonomo ${ }^{52}$. Adesso siamo pronti a esplicitare nella sua interezza l'abbreviazione «poliarchia elettorale ». La definizione (descrittiva) completa che propongo è la seguente: la democrazia è una procedura e/o un meccanismo che a) genera una poliarchia aperta, la cui competizione sul mercato elettorale $b$ ) conferisce potere al popolo e $c$ ) specificamente induce $\mathrm{i}$ governanti alla ricettività verso i governati.

Siamo cosí giunti al problema successivo: aumentare la « democraticità » della democrazia, e precisamente portarla oltre quel tanto di democrazia reale (non fittizia) a cui ci lascia il meccanismo competitivo. La democratizzazione della poliarchia, come vorrebbe Dahl, avviene congiuntamente, anche se non contemporaneamente, lungo due direttrici: 1) liberalizzazione e/o contestazione pubblica e 2) inclusività e/o partecipazione ${ }^{53}$. Chiariamo subito che Dahl concepisce questo tracciato e le sue categorie come le «direzioni » lungo le quali ogni e qualsiasi regime cambia e alla fine si trasforma in un altro. Se si limita il problema alle poliarchie, a mio giudizio «partecipazione » va

52 Questo è un punto del tutto trascurato dagli anti-elitisti. La loro tesi, in genere, è che la maggioranza del popolo rimane senza 'voce', dato che non può organizzarsi in gruppi di pressione. Il fatto che le maggioranze proprio in quanto maggioranze elettorali, abbiano una 'voce', che spesso prevale sulle altre pressioni viene costantemente (e significativamente) ignorato.

53 Cfr. Dahl, Polarcby, cit., pp. 4-8. 
meglio di « inclusività ${ }^{54} \mathrm{e}$ 《liberalizzazione » va meglio di « contestazione ${ }^{55}$. Comunque, questa traccia basta a indicare le direzioni lungo le quali Dahl affronta il problema nell'ambito della sua teoria della poliarchia. Quanto a me, vorrei limitarmi a fissare i seguenti punti: 1) ciò che rende possibile la democrazia non va confuso con quanto la rende piú democratica; 2) se non si trattano i due problemi esattamente in quest'ordine, i buoi rischiano di danneggiare il carro invece che tirarlo; 3) la teoria competitiva della democrazia, una volta stabilita, può curarsi, e lo fa, di massimizzare la democrazia, cosí adeguandosi anche a quanto predicava la teoria classica. E questo mi porta al problema finale: definire la democrazia, come sistema di governo, dal punto di vista prescrittivo.

\section{Definizione normativa della poliarcbia}

Dal punto di vista descrittivo, ho detto, la democrazia è una poliarchia elettiva. Ma come dovrebbe essere? Poiché la poliarchia è uno stato de facto, qual è il suo corrispondente stato de iure? Come si sa, il problema è se la democrazia rappresentativa sarà in grado, a lungo andare, di funzionare, e magari di migliorare le sue prestazioni, senza una pressione di valore sua propria, e anzi di fronte a una pressione di valore che sempre piú - e ora in modo schiacciante - svaluta la dimensione verticale.

Tale svalutazione appare chiara, ed è testimoniata, dalla situazione attuale del nostro lessico. Le parole che tipicamente fanno riferimento alla dimensione verticale sono « elezione », «élite » e «selezione ». Tutti questi termini sono stati conce-

54 Questa interpretazione è convalidata da due motivi. Primo, è Dahl che rileva come la « inclusività » da sola porti a una « egemonia chiusa », cioè a regimi plebiscitari e di mobilitazione. Secondo, quando Dahl parla specificamente di «buona società » (in After the Revolution?, New Haven, Yale University Press, 1970) si riferisce in complesso, al problema della partecipazione.

55 Specificamente, la mia difficoltà con il termine 'contestazione' viene dal fatto che la fattispecie non supera la prova del principio del pericolo opposto. (Cfr. Democratic Theory, cit. cap. V, sez. 5). La contestazione è una forza democratizzante fino a che si oppone a una oligarchia; ma non oltre. Direi, dunque, che per una poliarchia va meglio « voce »; vedi A. O. Hirschman, Exit, Voice, and Loyalty, Cambridge, Mass., Harvard, University Press, 1970. 
piti con connotazioni valutative. Elezione ha indicato, per circa quindici secoli, una scelta qualitativa. Si pensi a «gli eletti » che nel linguaggio protestante sono i chiamati da Dio. Elite deriva dalla stessa radice, e fu coniata - quando « aristocrazia » perse il suo significato originale e passò a designare soltanto un ceto - appunto per indicare «il meglio », gli aristói, la parte scelta. Selezione deriva, invece, da seligere, ma gradatamente è andata allineandosi con eligere (quando elezione è passata a designare il mero atto di votare) nel significare una scelta basata sulla eccellenza o sulla congruità.

Nell'odierno linguaggio politico tutte queste connotazioni sono perdute o comunque sotto attacco. Elezione è ridotta a un solo significato: il puro e semplice atto di votare. Selezione indica poco piú di una semplice preferenza della volontà, quando non viene addirittura distorta e screditata come «discriminazione ». Di conseguenza nel nostro linguaggio «gli eletti » sono soltanto coloro che sono stati votati per una determinata carica; e affermare che gli eletti dovrebbero essere «selezionati » suona piú come una ridondanza che come una specificazione assiologica. Elite, infine, si è trasformata prima in una parola neutrale e poi, con gli attuali anti-elitisti, in un termine spregiativo. In entrambi i casi, élite - in totale oblio e negazione della sua ragion d'essere - viene associata con i potenti e/o i privilegiati.

Le suindicate trasformazioni possono essere attribuite a quella corruzione politica del linguaggio ben denunciata, fra gli altri, da Orwell ${ }^{56}$. Ma se anche ciò è vero in ordine agli eccessi recenti, le trasformazioni suddette rispecchiano, in generale, la visione e «valorizzazione » orizzontali della politica. In entrambi i casi, comunque, ci troviamo davanti a un circolo vizioso, che va accelerandosi fino a diventare un vortice vizioso. Senza connotazioni di valore otteniamo un vuoto di valori; e quando una parola buona si muta in una cattiva ci resta un vocabolario che sa esprimere soltanto negazioni. In questo modo si finisce anche - per mancanza di parole usabili - per rimuovere $\mathrm{i}$ problemi della « visibilità »: e quando un problema non si vede piú, esso sfugge al controllo. Perciò, per sfuggire al vortice vizioso, dobbiamo ripristinare il linguaggio che consente di «tornare a vedere » il nostro problema.

56 Vedi G. Orwell, Politics and the English Language, ora in Collected Essays, Harmondsworth, Penguin, 1957. 
Cominciamo con «selezione ». Qui si tratta di una causa tutt'altro che persa, perché è soltanto in politica - ed è un fatto rivelatore - che il termine è stato neutralizzato. Molti di coloro che usano elezione e selezione come termini intercambiabili passano automaticamente, in tutti i campi «non-politici », al significato valutativo del termine. Una impresa scientifica deve, per essere tale, selezionare il suo personale. Un'azienda che assuma personale, $\mathrm{o}$ lo seleziona $\mathrm{o}$ finirà probabilmente in fallimento. Forse che la politica della democrazia è tanto semplice, e tanto intrinsecamente diversa rispetto a tutti gli altri processi sociali, da rendere la selezione superflua o addirittura iniqua? Se cosí non è, allora la mia prima definizione in chiave assiologica sarà: «la democrazia dovrebbe essere un sistema selettivo di minoranze elette in competizione ». In breve, e in simmetria con la nostra precedente definizione descrittiva, « la democrazia dovrebbe essere una poliarchia selettiva ». Ma merita affrontare il problema di petto, e cioè dall'angolo visuale dell'eguaglianza.

Se l'eguaglianza - come ho detto prima - è la bussola della democrazia, è una bussola senza nord e sud: si muove solo verso l'est o l'ovest. Possiamo dire, perciò, parafrasando Marcuse, che siamo ridotti alla «eguaglianza ad una dimensione ». $\mathrm{Ma}$ vediamo meglio, facendo di nuovo riferimento a Dahl. Il primo Dahl contempla una classe di poliarchie che chiama «poliarchie egualitarie ${ }^{57}$. In passato ho espresso delle critiche a questo concetto ${ }^{58}$. Rivedendolo ora, mi ravvedo: può essere intéso come un modo di collegare gli elementi descrittivi (poliarchia) e di valore (eguaglianza). Comunque, Dahl non persegue questo spunto e, quando torna sull'argomento, sostiene che nei grandi sistemi «l'eguaglianza di potere» deve mutarsi, su basi di realismo, in « eguaglianza di opportunità » ${ }^{59}$. In termini operazionali questo aggiustamento di tiro è corretto. Eppure, se l'eguaglianza di opportunità giustifica i processi verticali della democrazia, la sua prospettiva di valore è ancora orizzontale: riguarda l'inizio. Una eguaglianza di opportunità

57 R. A. Dahl, A Preface to Democratic Theory, Chicago, The University of Chicago Press, 1956, p. 87.

58 Nella prima edizione di questo libro; cfr. Democratic Theory, New York, Praeger, 1965. Nel corrispondente capitolo della nuova edizione ho rivisto molte delle mie prime critiche a Dahl.

${ }_{59}$ Cfr. R. A. Dahl, Power, Pluralism and Democracy: A Modest Proposal; APSA paper, 1964. 
giustifica il fatto di « emergere », ma non valida, né attribuisce valore, a tale emergere.

Per concepire, quindi, l'eguaglianza come un valore innalzante, cioè come un valore teso verso l'alto, dobbiamo andare oltre l'eguaglianza di opportunità e giungere alla « eguaglianza di merito $»$, o nel merito. In tale prospettiva, e in armonia con essa, la definizione sarà: « la democrazia dovrebbe essere una poliarchia di merito ». Infatti l'asserzione che siamo « eguali nel volere » si applica solo alla dimensione orizzontale. Nella dimensione verticale possiamo essere eguali soltanto nel talento, nella competenza e nella rispondenza ai compiti richiesti. In sede verticale rendere eguali $\mathrm{i}$ diseguali non ha né senso né giustificazione. Considerare eguali talenti diseguali non rappresenta un'eguaglianza giusta, ma un'eguaglianza ingiusta e, per di piú, socialmente costosa e dannosa.

Quanto sopra si colloca ad un alto livello di astrazione, mentre l'eguaglianza, nella vita reale, va ridotta a domande molto concrete: egúale rispetto a cosa? e/o eguale rispetto a chi? Questa ultima è la domanda che ci interessa, visto che qui eguaglianza è riferita a un «gruppo di riferimento ». Con il che ricompare, inevitabilmente, « élite » nel suo significato originale ${ }^{60}$. Dico inevitabilmente per piú ragioni. Perché il termine élite è l'equivalente moderno degli antichi concetti di melior pars e sanior pars. Perché abbiamo bisogno di distinguere tra fatto e merito, fra chi è al potere (la classe politica di fatto) e chi merita il potere (le potenziali élites politiche). Infine, perché non abbiamo scelta, dato che il vocabolario politico non dispone di altri termini per rendere l'idea di capacità e di eccellenza.

Il punto è che, nel dare l'idea di « degno di scelta », il termine élite denota un gruppo di riferimento, e precisamente un gruppo di riferimento di valore. La domanda era: eguale rispetto a chi? La risposta è: rispetto all'élite come «parametro di valore ». Il sottinteso è che le élites reali non sono composte da coloro che si trovano al potere, né con loro coincidono. Al contrario, nell'ottica del gruppo di riferimento i gruppi concreti sono sottoposti a esame costante: essi «valgono » in base alle loro virtú, e solo se possiedono delle virtú.

$60 \mathrm{Si}$ osservi che, esattamente come nel caso di « selezione », il termine élite è stato svalutato solo nella sfera della politica. Quando parliaino, ad esempio, di élites intellettuali la connotazione originale rimane. 
Possiamo dire perciò: l'eguaglianza sollecita concretamente un elevamento, una salita di valore, quando viene staccata da «massa » e connessa invece a «élite ». $\mathrm{E}$ in questo modo la teoria del « rifarsi alle élites » (se cosí posso dire) fornisce alla « eguaglianza unidimensionale » una bussola completa, o completata.

Riassumiamo. Definendo la democrazia una poliarchia elettiva non ci occupiamo del « buon » funzionamento del sistema, perché la competizione elettorale non garantisce la qualità dei risultati, ma soltanto il loro carattere democratico. Il resto - il valore del risultato - dipende dalla qualità (non solo dalla ricettività) della leadership. Ma mentre il ruolo vitale della leadership viene spesso riconosciuto, ciononostante esso non ha una parte, o ha solo una parte trascurabile, nella teoria della democrazia. La mia ricerca di una definizione normativa « verticale » riguarda appunto questo problema. A tale scopo ho proposto una teoria del « riferimento alle élites » e due definizioni sommarie e tra di loro complementari: che la democrazia dovrebbe essere 1) una poliarchia selettiva e 2) una poliarchia di merito. Quale che sia il pregio di tali proposte, resta da constatare che la teoria generale della democrazia ha finora trascurato e mancato di indicare la proiezione di valore della eguaglianza nella dimensione verticale. Il che ci lascia non solo con una impoverita eguaglianza unidimensionale, ma anche, al tirar delle somme, con una democrazia unidimensionale, precaria e insoddisfacente.

Se fosse possibile una società acefala, potremmo rallegrarci: negli ultimi tempi in questo senso siamo in progresso. Ma se l'essere senza testa, o senza leaders, porta piú a una dissoluzione che a una soluzione, allora la denigrazione o timore delle élites propagandata dagli anti-elitisti costituisce un anacronismo che ci rende ciechi ai problemi non soltanto del futuro, ma anche del presente. Quanto piú perdiamo di vista la democrazia come forma di governo, tanto piú i nostri problemi si aggravano, e tanto piú diventano di quotidiano assillo. 